\title{
THE MASS DISTRIBUTION IN THE GALACTIC CENTER
}

\author{
M. T. McGINN, K. SELLGREN, E. E. BECKLIN and D. N. B. HALL \\ University of Hawaii
}

We present the results of a project to map the profile of the $2.3 \mu \mathrm{m} \mathrm{CO} \mathrm{V}=2-0$ bandhead in the integrated starlight in the central $10 \mathrm{pc}$ of the Galaxy. This is the first detailed determination of the kinematics of the faint stars that are the major constituent of the mass of the stellar cluster. The stars exhibit systematic rotation in the same sense as Galactic rotation, with $V_{1 S R}$ increasing with Galactocentric radius. The stellar velocity dispersion generally dominates the rotation and shows clear evidence for a radial gradient, in the sense of $\sigma$ decreasing with Galactocentric radius. The data are consistent with the dynamical center of the Galaxy being located at IRS 16 (to an accuracy of \pm 10 "). The mass distribution has been derived via the theory of stellar hydrodynamics and is shown in Fig. 1. For an assumed core radius of $10^{\prime \prime}(\sim 0.4 \mathrm{pc})$, the best fitting model is a combination of a central mass of $2.5 \times 10^{6}$ $M_{\odot}$ and a stellar cluster with a density dependence of $\mathbf{r}^{-2.1}$ (Fig. 1). If the core radius is small $\left(\sim 1^{\prime \prime}\right)$ then the mass distribution could be just due to a stellar cluster; a central condensed mass is not required to model the data in that case.

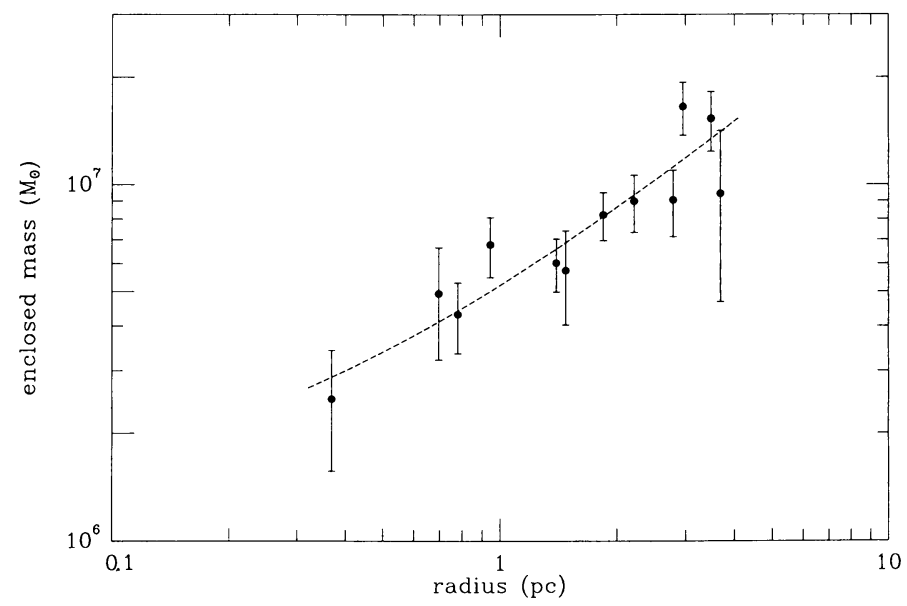

Figure 1: The mass distribution in the Galactic Center, derived from observations of the kinematics of the integrated starlight (McGinn, M. T., Sellgren, K., Becklin, E. E. and Hall, D. N. B. 1989, Ap. J., in press). The dashed line is a model fit for a core radius of 10", a central mass of $2.5 \times 10^{6} \mathrm{M}_{\odot}$ and a stellar cluster with a density dependence of $r^{-2.1}$. 